\title{
Toward understanding patient experience in idiopathic pulmonary fibrosis
}

\author{
Sonye K. Danoff \\ Affiliation: Division of Pulmonary and Critical Care, Johns Hopkins University School of Medicine, Baltimore, \\ MD, USA.
}

Correspondence: Sonye K. Danoff, Johns Hopkins University School of Medicine, 1830 E. Monument St., 5th Floor, Baltimore, MD 21205, USA. E-mail: sdanoffajhmi.edu

@ERSpublications

Validation of patient-reported outcomes in IPF is essential in incorporating patient voice in IPF clinical trials http://ow.ly/hyNM306jeGR

Cite this article as: Danoff SK. Toward understanding patient experience in idiopathic pulmonary fibrosis. Eur Respir J 2017; 49: 1602202 [https://doi.org/10.1183/13993003.02202-2016].

Idiopathic pulmonary fibrosis (IPF) is a progressive fibrotic lung disease which typically presents in the 6th or 7th decade of life with dyspnoea on exertion, cough and fatigue [1]. Based on a recent systematic review [2], global IPF incidence is 3-9 cases per 100000 per year in Europe and North America with increasing incidence over time. A similar incidence, 9 cases per 100000 , was reported in Canada using a narrow definition of IPF [3]. The age-adjusted mortality rate for IPF ranges from 2 to 10 per 100000 , resulting in an estimated 30000-60000 deaths in Europe in 2014 [4]. Despite the large number of individuals impacted by this nominally rare disease, there has been only one intervention proven to increase life expectancy in IPF and that is lung transplantation [5]. The small number of lung transplants available, and the common comorbidities of aging which accompany IPF, make this an inadequate intervention for the majority of patients. Unfortunately, over the past 40 years, multiple clinical trials in IPF have failed to achieve pre-specified primary outcomes.

On May 29, 2014, the prospects for patients with IPF in the United States and their caregivers changed dramatically with the publication of successful pivotal trials for two IPF therapies: nintedinib and pirfenidone [6,7]. These trial results were followed on October 15, 2014 by US Food \& Drug Administration (FDA) approval of both agents for the treatment of IPF. Pirfenidone had previously been approved in Europe, Canada and Asia based on the CAPACITY trial [8]. The basis for approval in all of these trials was the attainment of a surrogate endpoint: change in forced vital capacity (FVC).

The choice of a surrogate endpoint reflects the lack of a direct clinical endpoint. Although there has been some debate about using mortality as an endpoint, this has not risen to the level of a primary endpoint based on the number of patients needed to power such a trial [9]. In this context, the FDA has, along with many in the field, sought to promote more patient-focused outcomes (so called PROs). On the whole, the symptom-based instruments in common use are derived from other forms of chronic lung disease, such as chronic obstructive pulmonary disease (COPD). As such, their relevance in IPF clinical trials is not clear and, in the absence of a validated IPF specific PRO, efforts are being made to assess the properties of the COPD-derived PROs in the IPF patient experience.

The St George's Respiratory Questionnaire (SGRQ) is a PRO developed in 1992 for the assessment of health-related quality of life in obstructive lung disease [10]. It has been widely applied in COPD, asthma and bronchiectasis, and has been used as a secondary endpoint in multiple IPF clinical trials. The SGRQ is scored out of 100 with a higher score indicating poorer health-related quality of life. In addition to a total

Received: Nov 082016 | Accepted: Nov 092016

Conflict of interest: None declared.

Copyright OERS 2017 
score, scores in three domains: symptoms, activity and impact, are calculated. In this issue of the European Respiratory Journal, SwIGRIs et al. [11] make use of the data from a completed clinical trial of nintedinib in IPF (TOMORROW). The psychometric qualities of the SGRQ were evaluated in this well-defined IPF population. A high Cronbach's alpha score for the total, activity and impact scores showed a high level of internal consistency, suggesting acceptable reliability. Similarly, test-retest characteristics were acceptable. In considering the validity of the instrument, the SGRQ showed moderate consistency with Medical Research Council (MRC) dyspnoea scales. Notably, while some SGRQ subscales demonstrated moderate correlation with 6-min walk distance (6MWD), correlation with symptoms scores was weak. Similarly, in cross-sectional analysis, the SGRQ showed weak correlation with FVC and with diffusing capacity of the lung for carbon monoxide (DLCO). The mean SGRQ total score was higher in patients with lower lung function; however, the SGRQ showed only weak responsiveness to change in pulmonary function over 52 weeks.

Why does this matter? Well, the field of IPF therapy is rapidly expanding, with over 100 clinical trials currently registered in the United States on clinicaltrials.gov, and there is increasing evidence that mortality is unlikely to be an attainable endpoint for most clinical trials. Use of pulmonary function tests (PFTs) is a convenient surrogate but lacks the desired association with patient symptoms. Furthermore, slowing the decline in FVC can hardly be considered an outcome with relevance to most patients. Thus, the assessment of currently available PROs in the realm of IPF is necessary. While IPF-specific PROs are being tested, none are currently accepted by the FDA as outcome measures for clinical trials. Although the SGRQ addresses many of the common symptoms, including dyspnoea and cough, the current study suggests that it fails to demonstrate robust association with several clinically meaningful outcomes.

Perhaps more important than the evaluation of the SGRQ, this manuscript demonstrates the critical fund of knowledge that is available in completed clinical trials. In the current research climate, the idea of developing a 400-patient cohort for the purpose of testing PROs is virtually unthinkable. Yet within the context of multiple completed and ongoing clinical trials is the infrastructure for meaningful assessment of PROs in well-defined IPF populations. In light of the efforts by the FDA and other regulatory agencies worldwide to engage PROs as clinical trial outcomes, it seems prudent to use each clinical trial as an opportunity to evaluate the properties of both existing and new instruments.

\section{References}

1 Raghu G, Collard HR, Egan JJ, et al. An official ATS/ERS/JRS/ALAT statement: idiopathic pulmonary fibrosis: evidence-based guidelines for diagnosis and management. Am J Respir Crit Care Med 2011; 183: 788-824.

2 Hutchinson J, Fogarty A, Hubbard R, et al. Global incidence and mortality of idiopathic pulmonary fibrosis: a systematic review. Eur Respir J 2015; 46: 795-806.

3 Hopkins RB, Burke N, Fell C, et al. Epidemiology and survival of idiopathic pulmonary fibrosis from national data in Canada. Eur Respir J 2016; 48: 187-195.

4 Hutchinson JP, McKeever TM, Fogarty AW, et al. Increasing global mortality from idiopathic pulmonary fibrosis in the twenty-first century. Ann Am Thorac Soc 2014; 11: 1176-1185.

5 Thabut G, Mal H, Castier Y, et al. Survival benefit of lung transplantation for patients with idiopathic pulmonary fibrosis. J Thorac Cardiovasc Surg 2003; 126: 469-475.

6 Richeldi L, du Bois RM, Raghu G, et al. Efficacy and safety of nintedanib in idiopathic pulmonary fibrosis. $N$ Engl J Med 2014; 370: 2071-2082.

$7 \quad$ King TE Jr, Bradford WZ, Castro-Bernardini S, et al. A phase 3 trial of pirfenidone in patients with idiopathic pulmonary fibrosis. N Engl J Med 2014; 370: 2083-2092.

8 Noble PW, Albera C, Bradford WZ, et al. Pirfenidone in patients with idiopathic pulmonary fibrosis (CAPACITY): two randomised trials. Lancet 2011; 377: 1760-1769.

9 King TE Jr, Albera C, Bradford WZ, et al. All-cause mortality rate in patients with idiopathic pulmonary fibrosis. Implications for the design and execution of clinical trials. Am J Respir Crit Care Med 2014; 189: 825-831.

10 Jones PW, Quirk FH, Baveystock CM, et al. A self-complete measure for chronic airflow limitation-the St George's Respiratory Questionnaire. Am Rev Respir Dis 1992; 145: 1321-1327.

11 Swigris JJ, Esser D, Wilson H, et al. Psychometric properties of the St George's Respiratory Questionnaire in patients with idiopathic pulmonary fibrosis. Eur Respir J 2017; 49: 1601788. 